\title{
Role of robot assisted microsurgery in Plastic Surgery
}

\author{
S. Saraf \\ Department of Plastic Surgery, NMC Specialty Hospital, Dubai, UAE
}

Address for correspondence: Sanjay Saraf, Department of Plastic Surgery, NMC Specialty Hospital, Dubai, UAE. E-mail: drsaraf@hotmail.com

\section{ABSTRACT}

The application of telerobotics in the biomedical field has grown rapidly and is showing very promising results. Robot assisted microsurgery is one of the latest innovations of telerobotics in the field of Plastic surgery. The purpose of this article is to update the plastic surgery community on the expanding field of surgical robotics with an attempt to analyze various aspects related to human versus robotic assisted microsurgery.

\section{KEY WORDS}

RAMS, robot assisted microsurgery

\section{INTRODUCTION}

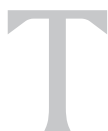

he essence of plastic surgery is to have innovative thinking, capacity to find new methods and to adopt newer technologies to its benefit. In the last decade, surgery and robotic technology have reached a maturity that has allowed them to work symbiotically to perform highly demanding surgeries. As microsurgical practice has begun to challenge the limits of human precision, our interest was to find out how microsurgery can be made more easier, more ergonomic and more predictable. Recent developments in the field of robot assisted microsurgery (RAMS) has promised encouraging results with this technology. RAMS is basically an advanced application of telerobotic surgery, as an answer to the surgeon's demand for ways to overcome the surgical limitations of microsurgery. RAMS allows performing high dexterity microsurgical operations with the help of robotic arms. The surgeon actually does a better, more precise, dexterous and highly controlled microsurgical procedure under high magnification resulting into optimal microsurgical outcome.

\section{REVIEW OF LITERATURE}

Though, initially the concept looked like science fiction, the robotic surgical tool was first developed for space use which later graduated as a precision instrument for microsurgery.

In the early 1990's, NASA's jet propulsion laboratory (JPL) began a project in telerobotics as part of its emergency response robotic program. The primary aim was to develop a robotic system (HAZBOT) to allow safe exploration of potentially dangerous sites (defusion of bombs, nuclear warfare, battle sites) and handling of hazardous materials (wastes from nuclear reactors). ${ }^{[1]}$ The engineers from NASA and the JPL also intended this for tele surgery in space to enable surgeons on earth to operate on astronauts at the space station. The time lag, however, prevented this from becoming feasible. The procedure was subsequently accelerated by various concomitant developments in computer technology and surgical related advancements. However, for a long time, placing dexterity enhancing robotic systems in the 
operating room remained an elusive goal. The subsequent evolution of the robotic surgical system culminated in the development of a different skill and advanced instrumentation. In the mid 90's there was a sudden surge in the development of robotic surgical technology. Dr. Alan Richards became the first surgeon to operate using the robotic system by performing a laparoscopic cholecystectomy that involved 25 minutes on the console. While the early writing on the new technology covered a variety of surgical procedures, special attention was given to cardiovascular procedures. In the mid nineties, Steve Charles originated the concept of a telerobotic system as a tool to assist the microsurgical procedures. ${ }^{[2]}$ Subsequently, in 1994-95 JPL engineers developed RAMS based on surgical requirements provided by Steve Charles using previously developed NASA telerobotics technology. It was a sixdegrees-of-freedom surgical robot slave made up of a torso-shoulder-elbow body with a three-axis wrist. The robot manipulator was about 10 inches long and 1 inch in diameter. ${ }^{[2]}$

Further review of the literature regarding RAMS revealed that most published articles appeared relatively recently, particularly after 1998 .

The two pioneering companies making surgical robots were the Computer Motion, founded in 1989 and Intuitive Surgical, formed in 1995..$^{[3]}$ Computer Motion developed Zeus robotic surgical system for minimally invasive microsurgery procedures and the Intuitive Surgical developed the daVinci surgical system consisting of a surgeon's console, a patient-side cart, a highperformance vision system and proprietary instruments and later added endowrist technology to the same. ${ }^{[4]}$ The Computer Motion and Intuitive Surgical companies finally merged into a single company, Intuitive Surgical in 2003.

In 1998, a study by Stephenson first pointed out to the fact that coronary artery anastomoses are technically feasible with the use of robotic instruments. ${ }^{[5,6]}$ An additional study done by the same group reported the successful use of this approach in a large animal trial. ${ }^{[7,8]}$ Further studies of the feasibility of endoscopic cardiac surgery was performed by various surgical teams verifying that robotic technology could be used to accomplish a completely endoscopic anastomosis. ${ }^{[9-11]}$ Additional studies involving cardiac procedures have also produced positive findings with regard to the clinical efficacy and benefits of robotic assisted anastomosis. In 1999, Schueler performed the world's first closedchest multivessel cardiac bypass using the daVinci system. ${ }^{[4]}$ In 1999, Neuromatic from Integrated Surgical System (ISS), Davis, California was cleared by FDA for use in stereotactic brain surgery. Mohr et al first used the da Vinci Robotic system and the AESOP system for ITA harvesting and CABG surgery. ${ }^{[12]}$ On July 11, 2000, FDA approved the first completely robotic surgery device, the da Vinci surgical system from Intuitive Surgical to perform general surgical procedures while seated at a computer console and 3-D video imaging system across the room from the patient. ${ }^{[4]}$ In 2000 , a German study found out that using the daVinci system to perform endoscopic beating heart (single or double) bypass surgery is safe, causes significantly less trauma to the patient and allows for quicker recovery.

In 2001, with a prototype RAMS, 10 carotid arteriotomies were created and closed using either the RAMS system or conventional microsurgical techniques. The precision, technical quality and error rate of telerobotic surgery were similar to those of conventional techniques but it was found to be associated with a twofold increase in the length of the procedure. ${ }^{[11]}$

An important comparative RAMS study was performed by several international scientific teams to analyze various features related to microvascular anastomosis. This comparative study was carried out between RAMS and surgeon performing anastomosis with 3-D endoscope. The mean total operative time per $3 \mathrm{~mm}$ robotic anastomosis, utilizing 9-0 suture using 2-D visual port was $29.5 \pm 15$ minutes (excluding setting up and dismantling robotic arms). The mean total operative time per $3 \mathrm{~mm}$ surgeon anastomosis using 3-D endoscope was found to be $16.3 \pm 5$ minutes. The inference was, though the robot took longer time for anastomosis, they performed high quality, tremor free precise microsurgery without any technological problem and intraoperative complications. ${ }^{|8|}$

\section{Technical details}

The typical surgical robot architecture follows a classical mater/slave tele-operation set up. This set up consists of two modules: the surgeon console (master) and the robot (slave). The surgeon's console is both viewing and active computer controlled console having set of 
handles, ergonomically designed along with integrated 3 -D vision system and in some cases voice command components. High resolution optical encoder is selected for transmitting the command from master arm to slave arm.

The robotic system interacting with the patient includes usually three robotic arms; two to manipulate the surgical instruments and a third to position the endoscopic camera at the optimal position. The surgeon controls the position of the robotic arms and in turn surgical instruments via handles at the console and third endoscopic camera arm by voice command, providing the surgeon precise and stable view of the actual surgical field.

\section{Configuration of RAMS}

Surgeon

- Surgeon's console (Master)

- Robotic arms (Slave)-2 arms

- Microsurgical instruments

Endoscopic camera

- Visualization of operating field

Robotic arm (voice activated)-3 $3^{\text {rd }}$ arm

- Endoscopic camera

Assistant/nurse

- For setting robotic arms

- For changing instruments

\section{DISCUSSION}

Telerobotics is a very important and quickly expanding field with faster processors and new algorithms, the development of telerobotic systems to assist surgeons is a growing field of research. In robotic telesurgery, the robotic tools are not automated robots but teleoperated systems under direct control of the surgeon. ${ }^{[13]}$

Robots are already being used in laparoscopic surgery, ${ }^{[14]}$ urological surgery, ${ }^{[15]}$ neurosurgery ${ }^{[11,16]}$ and cardiac surgery ${ }^{[6,7,12,17]}$ with varying success. They have been successful in orthopedic surgery to perform total hip arthroplasty surgery. ${ }^{[18]}$

This option of performing high precision surgery has sparked the potentially huge hope for its application in doing microsurgery in plastic surgery. Microsurgery is a specialized technique which requires many years of training to be proficient. In microsurgery, the instruments virtually become specialized extensions of the surgeon's hands. As the outcome in microsurgical procedures is often dependent on technical quality, the enhanced precision provided by robotic systems is likely to have profound effect on the outcome.

During microsurgery the surgeon has to manipulate tissue with the instruments and the result is likely to be the affected by individual surgeon's manual dexterity. In addition, several factors such as lengthy period, time constraint and tremors during the procedure can adversely affect the surgeon's technical performance. As the current microsurgical practice is now challenging the limits of human dexterity, stamina and patience, the limiting factors basically arise due to undesired involuntary and inadvertent movement of the hand which creates an error component in hand motion. ${ }^{[19]}$ The most familiar source of undesired hand motion is physiological tremor which is an approximately rhythmic, roughly sinusoidal involuntary component inherent in all human motion. ${ }^{[20]}$ Low frequency errors or drift are also present in hand motions and are often longer than tremos. ${ }^{[21]}$ Irregular high-frequency motions or jerk can also occur. ${ }^{[2]}$ The results are that some movements are less precise than is desired and some desired movements cannot be done at all. Microsurgical practice would therefore benefit greatly from RAMS that enhance accuracy by compensating position error.

RAMS is based on typical master slave tele-operation. Using RAMS, the surgeon sitting on the console orchestrates or commands the motions of the robotic arms to perform microsurgical procedures. The surgeons hand motions are transferred in a real-time through a computer system, where they are processed to automate the robotic movements. This process reduces the surgeon's movement at the tissue level and prevents tremor or inadvertent movement often associated with fatigue, anxiety, age related or other factors.

The advantages of RAMS are thus obvious. As hours of exacting work can tire anybody, superior ergonomics while seated at the console optimizes the surgeon's performance and dexterity. Any tremor in the surgeon's own hands and fingers is completely eliminated with the help of tremor filters and motion scalers leading to superior dexterity. ${ }^{[23]}$ Another important feature is that there is greatly increased precision due to scalability of 
movements which can be up to $1: 6$ scale, meaning that six $\mathrm{mm}$ movement of fingers will result in $1 \mathrm{~mm}$ movement of the instrument. ${ }^{[24]}$ This increased precision is of great importance during microsurgery, with complete elimination of hand and finger tremors.

Another feature is that we can always find the perfect angle towards the vessel due to enhanced rotating ability of the camera and wrists of the robotic arm. RAMS also provides more range of motion and more degree of freedom than the human hand leading to easy maneuverability in difficult positions. It can virtually be viewed as a specialized extension of the surgeon's hands. Other added features like optimal magnification with 3D visualization, superior resolution and 3-D spatial accuracy marks the characteristics of RAMS.

This indefatigable nature of RAMS is likely to be of enormous help in performing vascular anastomosis especially in cases of free flaps, digital replantations; microneurorrhaphy and other such demanding microsurgical procedures [Figure 1].

It also has a potentially invaluable use during microsurgery involving high risk patients /patients with HIV, to protect the surgeon from virus transmission.

Inspite of all mentioned advantages, there are some limitations also. The initial capital cost ranging from one million to several million dollars is prohibitive for its free use. However, multi-specialty utilization of robotic technology along with improvement in surgical outcome and more expeditious return to work will make this approach cost-effective, justifying investment in this

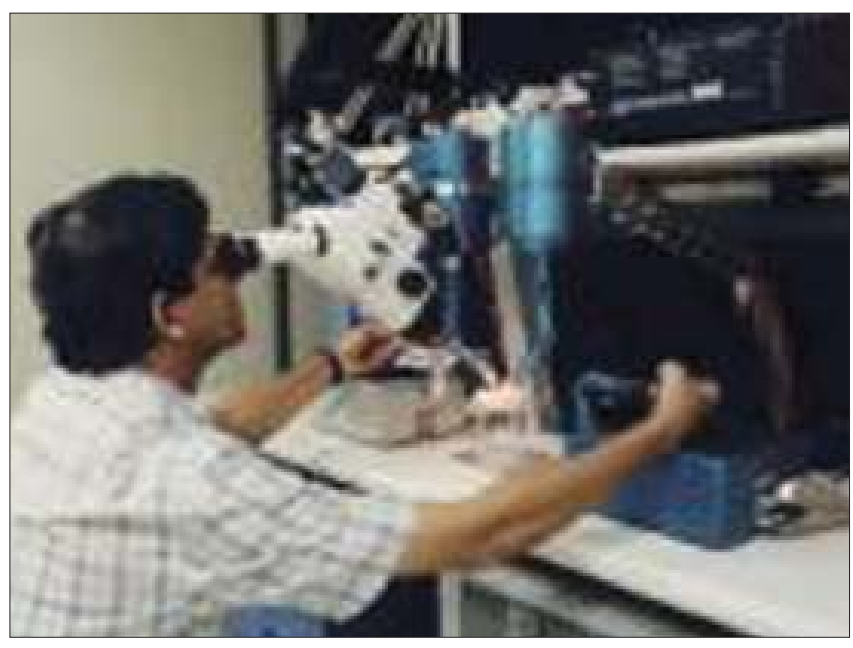

Figure 1: RAM Prototype technology. The time taken for the surgery is often more as compared to the conventional surgery. However, operating time is likely to reduce significantly with more familiarity and decreasing learning curve. A study by Siemionow et al concluded that concurrent use of the RAMS as a microsurgical assistant is applicable in microsurgery, with the advantages of greater precision and more rapid microsurgical manipulation. ${ }^{[25]}$ Another current limitation is that the presently available instruments are not yet small and fine enough to perform delicate micro-vascular surgeries like free flaps, microneurorrhaphy and digital replantations. ${ }^{[24]}$ Another limitation noted is that there is no haptic feedback which often make the surgeon feel detached from the patient and the procedure. However, high magnification of operative site negates this draw back. ${ }^{[24]}$ Also a learning curve is always there but after dedicated training and some experience, one feels comfortable working with the instrumentation and doing the surgery without actually touching the patient. Clearly, a lot of work needs to be done and the dividends are expected to be equally pleasing.

The term robotic surgery also probably gives an impression of a Robot independently operating on a patient in operation theatre. This image is not correct as they do not replace the surgeon at all in the operation theatre. They only maneuver the surgical instruments necessary for surgery and are always under the direct, total control of the surgeon. As JPL's Tom Hamilton rightly said "RAMS takes the most skilled surgeon and makes his or her skills better. RAMS can improve surgical techniques to allow faster and safer procedures".'26]

With further advances in areas of 3-D video imaging and display systems, tele-operative controls, telemanipulators, graphic planners and micro-tools, surgeon's capabilities will be tremendously increased with much improved surgical outcome. These advances will certainly make microsurgeries easier to perform, cost effective in the longer run and with further refinements, prove to be a dependable associate of plastic surgeons.

\section{CONCLUSION}

Surgical robotic technology is now on the cusp of revolutionizing microsurgical capabilities. With the latest advancements in the field of RAMS, the armamentarium 
available to the plastic surgeons will be greatly expanded. The advantages are self evident. The use of RAMS technology during microsurgery can greatly improve the microsurgical outcome by providing surgeons with greater precision, elimination of hand tremors, increased range of motion and enhanced 3-D visualization.

However, the robots are never likely to replace the highly evolutionized human hand and rather than replacing the human hand, this technology will help to retain the benefits of the human hand along with its superlative optimization to achieve the goal of optimal precision and predictability. With the continued evolution of robotic surgical technology, the robots are expected to become smaller, faster, lighter and smarter with exponential increased application in microsurgery. The future of RAMS seems to be promising and continuing advancement of this technology holds the key.

\section{REFERENCES}

1. Edmonds GO, Welch RV. "Applying Robotics to HAZMAT". Proceedings of the $4^{\text {th }}$ National Technology Transfer Conference and Exposition-In NASA Technology 2003. 1993;2:279-87. Available from: http://trs-new.jpl.nasa.gov/ dspace/bitstream/2014/35273/1/93-0854.pdf

2. Turner J, editor. Robotic Microsurgery to Make Difficult Procedures Easier. Aerospace Technology Innovation; $9^{\text {th }}$ ed: 1997. Available from: http://nctn.hq.nasa.gov/innovation/ Innovation52/robsurg.htm

3. Bushnell P. Robotic Surgery: A New Role for Machines in Medicine; Bon Secours Health System, Inc. TEWS- White Paper; July 2001. Available from: http://www.bshsi.com/tews/docs/ ROBOTIC\%20SURGERY.pdf

4. Schaff TA. Robotic Surgery: The Future is Now Telerobotic Application. 2001. Available from: http://www.devicelink.com/ mx/archive/01/03/0103mx024.html

5. Stephenson ER. Sankholkar S, Ducko CT, Damiano RJ. Robotically assisted microsurgery for endoscopic coronary artery bypass grafting. Ann Thorac Surg 1998;66:1064-7.

6. Stephenson ER, Sankholkar S, Ducko CT, Damiano RJ. Successful endoscopic coronary artery bypass grafting: An acute large animal trial. J Thorac Cardiovasc Surg 1998;116:1071-3.

7. Schenker PS, Das H, Ohm T. Development of a Master-Slave manipulator for Dexterity-Enhanced Microsurgery. Telemanipulator \& Telepresence Technologies, SPIE Proc. 2351: Boston, MA; 1994.

8. Schenker PS, Das H, Ohm TR. A new robot for high dexterity microsurgery", in Ayache, N. (editors), Computer Vision, Virtual Reality and Robotics in Medicine. Proceedings of 1995 Conference on Computer Vision, Virtual Reality, Springer-Verlag: Berlin, Germany; 15-22.
9. Szymula TP, Richter V. Recenzie Endoscopy and Microsurgery, D. Pieptu, In the series "Update in Plastic Surgery. Manfred Fray (ed) 2001, Spinger Wien New York. In Romanian Journal of Hand and Reconstructive Microsurgery: 2001. p. 1-2. Available from: http://www.rjhrm.ro/pdf/43.pdf

10. Schiff J, Li PS, Goldstein M. Robotic microsurgical vasovasostomy and vasoepididymostomy in Rats. Int $\mathrm{J}$ Med Robot Comput Assist Surg 1:122-6.

11. Le Roux, Peter D, Das H, Esquenazi S, Kelly J, Patrick MD. Robotic assisted microsurgery: A feasibility study in the Rat. Neurosurgery 2001;48:584-9.

12. Mohr FW, Falk V, Diegeler A, Autschbach R. Computer-enhanced coronary artery bypass surgery. J Thorac Cardiovasc Surg 1999;117:1212-4.

13. Cavusoglu MC, Williams W, Tendick F, Sastry SS. Robotics for telesurgery: Second generation Berkeley/UCSF laparoscopic telesurgical workstation and Looking towards the future applications. Industrial Robot 2003;301:22-9. Available from: http://www.emeraldinsight.com/Insight/View Content Servlet. Emerald Full Text Artice/Articles/0490300101.html

14. Marescaux J, Smith MK, Folscher D, Jamali F, Malassagne B, Leroy J. Telerobotic Laparoscopic Cholecystectomy: Initial Clinical Experience With 25 Patients. Ann Surg 2001;234:1-7.

15. Hoznek A, Zaki SK, Samadi B, Salomon L, Lobontiu A, Lang P, et al. Robotic Assisted Kidney Transplantation: An Initial Experience. J Urol 2002;167:1604-6.

16. Zimmermann M, Krishnan R, Raabe A, Seifert V. Robot-assisted Navigated Neuroendoscopy. Neurosurgery 2002;51:1446-52.

17. Tang LW, D'Ancona G, Bergsland J, Kawaguchi A, Karamanoukian HL. Robotically assisted video enhanced endoscopic coronary artery bypass graft surgery. Angiology 2001;52:99-102.

18. Taylor RH, Mittelstadt BD, Paul HA, Hanson W, Kazanzides P. An image-directed robotics system for precise orthopaedic surgery. IEEE Trans Robot Auto 1994;10:261-75.

19. Riviere CN, Khosala PK. Active hand-held instrument for error compensation in microsurgery. Proceedings of Intelligent System and Manufacturing: Tech. Conf. on Microrobotics and minisystem fabrication: Pittsburg; 1997. p. 86-95.

20. Elble RJ. Central Mechanisms of Tremor. J Clin Neurophysiol 1996;13:133-44.

21. Riviere CN, Radar RS, Khosla PK. Characteristics of hand motion of eye surgeons. Proc. $19^{\text {th }}$ Annual Conference of the IEEE Engineering in Medicine and Biology Society: 1997. p. 1690-3.

22. Schenker PS, Barlow EC, Boswell CD, Das H, Lee S, Ohm TR, et al. Development of a telemanipulator for dexterity enhanced microsurgery. Proc $2^{\text {nd }}$ Intl Symp Med Robot Comput Assist Surg 1995. p. 81-8. Available from: http://www.emeraldinsight.com/ Insight/ViewContentServletEmeraldFullTextArticle/Articles/ 0490240212.html

23. Louw DF, Fielding T, McBeth PB, Gregoris D, Newhook P, Sutherland GR. Surgical Robotics: A Review and Neurosurgical Prototype Development. Neurosurgery 2004;54:523-37.

24. Rosson GD. Robotic-Assisted Microsurgery. Reconst Microsurg Spring Summer 2005;16:17-8.

25. Siemionow M, Ozer K, Siemionow W, Lister G. Robotic assistance in microsurgery. J Reconstr Microsurg 2000;16:643-9.

26. Innovation-Robotic Microsurgery. Robotic Microsurgery to Make Difficult Procedures Easier; 1997. Available from: http:// ipp.nasa.gov/innovation/Innovation52/robsurg.htm 